\title{
Composite Nasopharyngeal plasmacytoma with nodal paraimmunoblastic variant of small lymphocytic lymphoma: A case report
}

\author{
KAREN NALBANDYAN ${ }^{1}$, DANIEL BENHARROCH ${ }^{1}$, ORY ROUVIO $^{2}$ and ANNA GUREVITCH ${ }^{2}$ \\ Departments of ${ }^{1}$ Pathology and ${ }^{2}$ Hematology, Soroka University Medical Center and Faculty of \\ Health Sciences, Ben Gurion University of The Negev, Beer-Sheva 84101, Israel
}

Received May 11, 2016; Accepted January 19, 2017

DOI: $10.3892 / \mathrm{ol} .2017 .6308$

\begin{abstract}
A 78-year-old male who presented with severe shortness of breath and bilateral nasal congestion was shown to exhibit ta mass localized in the nasopharynx. The tumor was diagnosed as an extramedullary plasmacytoma. Peripheral blood eosinophilia had been persistently noted in the preceding 12 years. The plasmacytoma exhibited a predominance of $\kappa$-light chain monotypic Mott cells and was admixed with numerous eosinophils. No history of allergic rhinitis, asthma or aspirin sensitivity was elicited. An axillary lymph node was excised two weeks after the nasopharyngeal biopsy, and it exhibited a paraimmunoblastic transformation of small lymphocytic lymphoma. A review of the literature identified few occurrences of such simultaneous tumors in individual patients.
\end{abstract}

\section{Introduction}

Extramedullary plasmacytoma is a relatively rare disease, characterized by a localized monoclonal plasma cell proliferation, in the absence of demonstrable systemic involvement. It comprises $5-10 \%$ of all plasma cell neoplasms. The most common sites of involvement are in the head and neck, particularly the upper respiratory tract (including the nasal cavity and the nasopharynx, which by itself is a rare occurrence) (1). To date, the origin of this type of tumor in chronic nasopharyngitis has not been described. Chronic nasopharyngitis has resulted in increased populations of eosinophils and has been associated with nasal polyps, allergic rhinitis, asthma and/or aspirin sensitivity (2). Paraimmunoblastic transformation rarely

Correspondence to: Professor Daniel Benharroch, Department of Pathology, Soroka University Medical Center, P.O. Box 151, 1 Itshak Rager Boulevard, Beer-Sheva 84101, Israel

E-mail: danielbenharroch1@gmail.com

Key words: synchronous, plasmacytoma, paraimmunoblastic transformation of small lymphocytic lymphoma develops in chronic lymphocytic leukemia/small lymphocytic lymphoma (CLL/SLL) (3).

The present study describes the simultaneous occurrence of a nasopharyngeal extramedullary plasmacytoma with the paraimmunoblastic transformation of a newly diagnosed small lymphocytic lymphoma. An association between the two malignant tumors is discussed.

\section{Case report}

A 78-year-old male was admitted to the emergency room at the Soroka University Medical Center (Beer-Sheva, Israel) in August, 2015, with dyspnea, dysarthria and nasal congestion. An ear, nose and throat examination associated the symptoms with a narrowing of the nasopharynx by a mass, bilateral tonsillar enlargement and cervical and axillary lymphadenopathy. In addition, a total body computed tomography (CT) scan revealed generalized lymphadenopathy without hepatosplenomegaly. A nasopharyngeal biopsy was performed under endoscopy in August, 2015. The tissue diagnosis of a $\kappa$-light chain plasmacytoma, rich in eosinophils, was established, as illustrated in Fig. 1A-C. Serum protein electrophoresis and immunofixation revealed a monoclonal spike of Immunoglobulin ( $\mathrm{Ig}$ )G-к. A bone marrow aspiration suggested a lymphoproliferative disorder, but no plasma cells were present. Mild to moderate peripheral blood eosinophilia had been present since 2003, but no allergic rhinitis, asthma, nasal polyposis or aspirin sensitivity were detected. As an immediate diagnosis of the nasopharyngeal lesion could not able be made, a left axillary lymph node excision was performed in October 2015.

The clinical diagnosis of the nasopharyngeal mass was based on an endoscopic examination of the nasopharynx and on a CT scan. From the nasopharyngeal biopsy, two fragments of tissue were obtained and the largest was $1.2 \mathrm{x} 1 \mathrm{~cm}$ in size.

The histopathological diagnoses were based on hematoxylin and eosin 5- $\mu$ m-thick stained sections of formalin-fixed paraffin-embedded tissue, according to standard protocols and immunohistochemistry using a Ventana BenchMark XT (Ventana Medical Systems, Tucson, AZ, USA). Immunostaining included cluster of differentiation (CD) 45 (Dako; Agilent Technologies, Inc., Santa Clara, CA, USA), CD20 


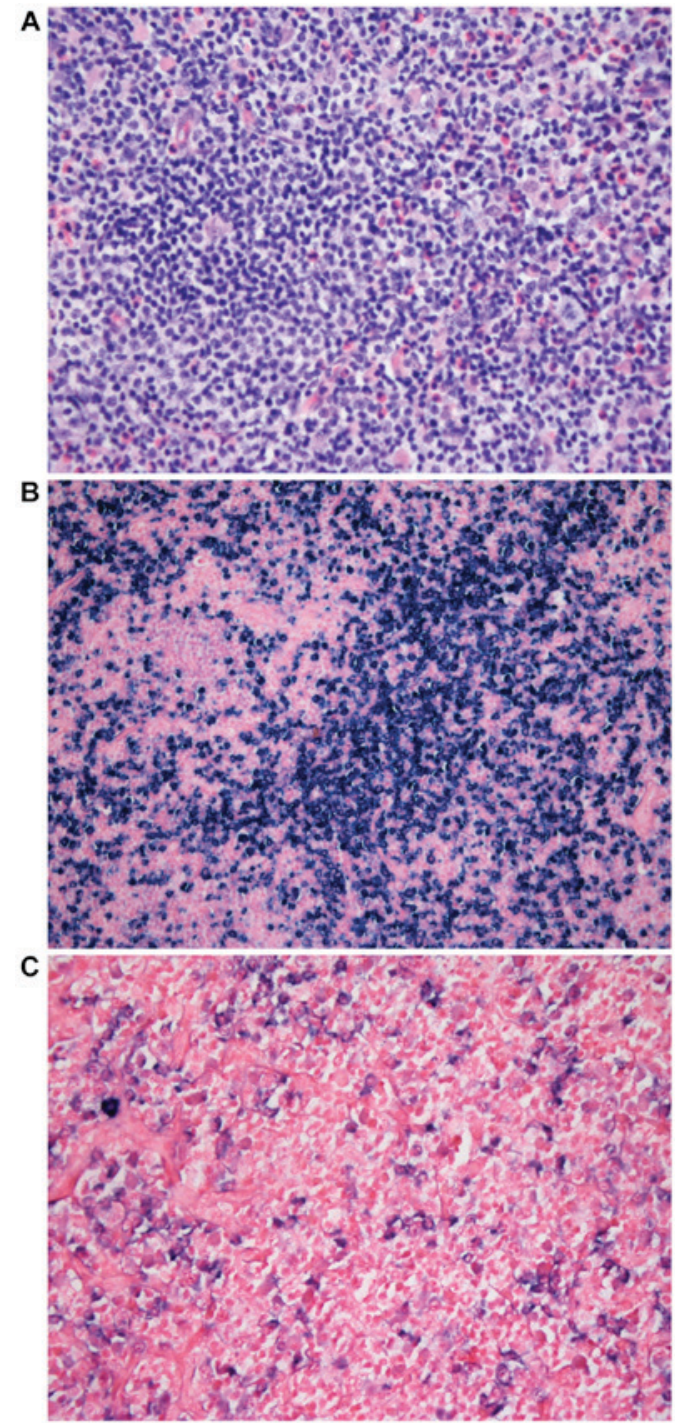

Figure 1. Nasopharyngeal biopsy. (A) Mixed cell infiltrate: Numerous plasma cells and Mott cells, eosinophils, small round lymphocytes and a number of immunoblasts, visualised with hematoxylin and eosin staining. Magnification, x340). (B) Numerous к-positive plasma cells (ISH; Ventana BenchMark). Magnification, x260. (C) Rare $\lambda$-positive plasma cells (IHC). Magnification, x260. IHC, immunohistochemistry.

(DakoAgilent Technologies, Inc), CD3 (Thermo Fisher Scientific, Inc., Waltham, MA, USA), CD5 (Novocastra; Leica Microsystems GmbH, Wetzlar, Germany), CD30 (DakoAgilent Technologies, Inc), CD15 (DakoAgilent Technologies, Inc.), CD23 (Novocastra; Leica Microsystems GmbH), and Ki-67 (Thermo Fisher Scientific, Inc.), alongside $\delta$-(Cell Marque; Sigma-Aldrich; Merck KGaA, Darmstadt, Germany) and $\kappa-(C e l l$ Marque; Sigma-Aldrich; Merck KGaA) Ig light chains. In addition, Ig light chain RNA was studied by in situ hybridization.

Histological examination using the Olympus BX41 microscope (Olympus, Tokyo, Japan) revealed that the tissue was hypercellular with prominent plasma cells, a majority of which were Mott cells. In addition, small to medium-sized lymphocytes, numerous eosinophils and a number of scattered large lymphoid cells, most probably immunoblasts, were identified.

The Mott cells and additional plasma cells were $\kappa$-light chain monotypic: CD138 moderate (++) and CD20 negative
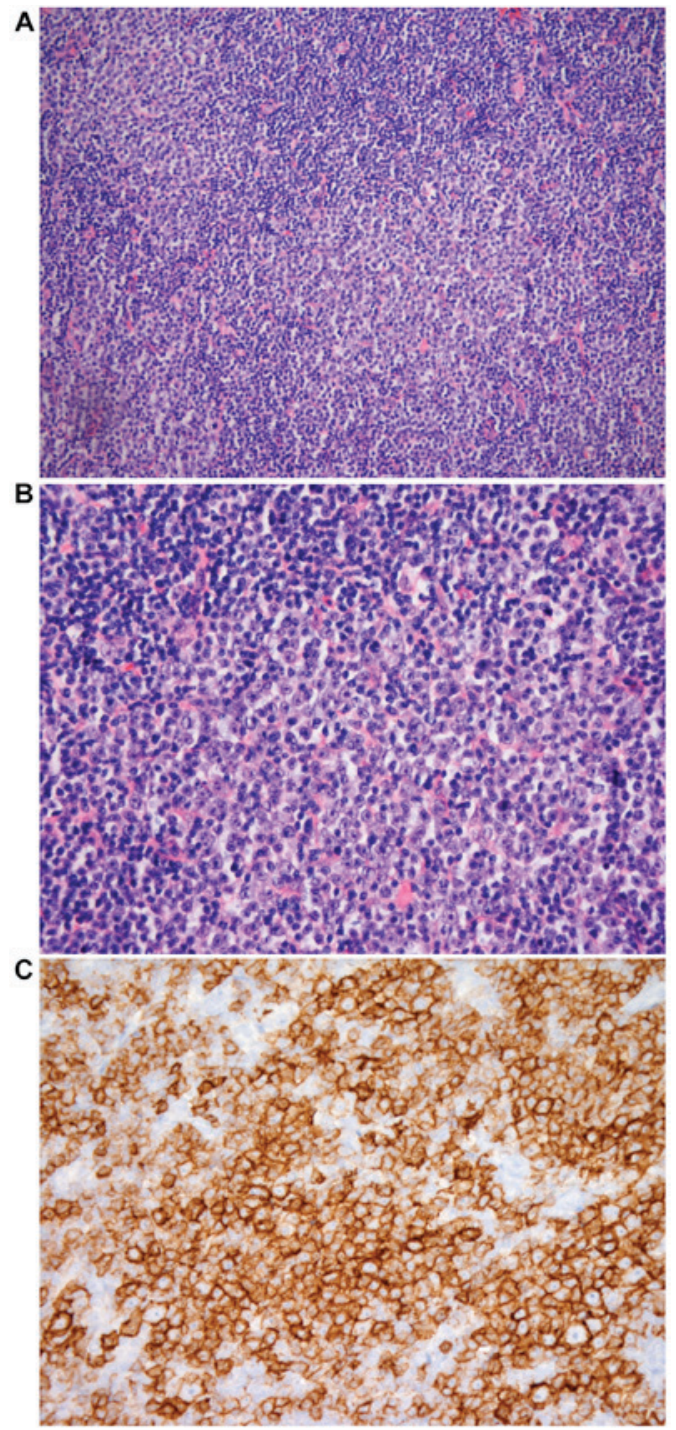

Figure 2. Sections from the axillary lymph node. (A) Vague nodularity highlighting large proliferation centres, often confluent, surrounded by small round lymphocytes. Stained with H\&E. Magnification, x260. (B) Higher magnification of a proliferation center, composed mainly of paraimmunoblasts. Several vesicular nuclei exhibit a central nucleolus. Stained with H\&E. Magnification, x340. (C) The paraimmunoblasts stain strongly for cluster of differentiation 20 (immunohistochemistry staining with 3,3'-diaminobensidine substrate; magnification, x340). H\&E, hematoxylin and eosin.

(-). The immunoblast immunostaining demonstrated a CD30 weak (+), CD15-, melanoma-associated antigen (mutated) 1 $(\mathrm{MUM1})^{+}$, B-cell-specific activator protein ${ }^{+}$faint, Epstein-Barr virus (EBV)/latent membrane protein $1^{-}$, EBV-encoded small $\mathrm{RNAs}^{+}$in a small number of cells, fascin ${ }^{+}, \mathrm{CD}_{2} 0^{+}$and $\mathrm{CD}^{-}$ profile. A nasopharyngeal extramedullary plasmacytoma was diagnosed.

The lymph node biopsy suggested areas consistent with the diagnosis of CLL/SLL with small round lymphocytes, $\mathrm{CD} 20^{+}$expression; $\mathrm{CD}^{+}$expression that was $>\mathrm{CD}^{+}$expression levels and $\mathrm{CD} 23^{++}$expression levels. Proliferation centers were prominent and often confluent. These areas contained almost exclusively a population of medium to large-sized lymphoid cells with clear cytoplasm, a vesicular and irregular nucleus and a small central eosinophilic nucleolus with several admixed eosinophils. The larger lymphoid cells 
exhibited strong (+++) expression levels of $\mathrm{LCA}^{+++}, \mathrm{CD} 20^{+++}$, $\mathrm{CD} 23^{+++}$and $\mathrm{CD}^{+++}$, which were $>\mathrm{CD}^{+}$expression, weak and cellular expression of $\mathrm{CD} 21^{+}$and weak expression of B-cell lymphoma $6^{+}$and $\mathrm{MUM1}^{+}$. The proliferation fraction of the proliferation centers (Ki-67) was $70 \%$. The diagnosis of a variant paraimmunoblastic transformation of CLL/SLL of the lymph node was made, as demonstrated in Fig. 2A-C. However, no clinical evidence for CLL was present.

The bone marrow biopsy was normocellular for the age of the patient. A near normal maturation of the hematopoietic elements was evident. A small lymphocytic aggregate, with small round lymphocytes, was isolated but was insufficient for the diagnosis of CLL/SLL. No plasmacytosis was observed, which confirmed the extramedullary nature of the plasmacytoma.

As the surgical resection was incomplete, the role of adjuvant radiotherapy for the nasopharyngeal tumor was not clear. However, radiotherapy was deemed necessary for the present patient $(4,5)$. The frequency and extent of the follow up assessment in these types of cases depends on the patient and the attending physician. During the visits, besides a physical examination, serum protein electrophoresis and immunofixation, a fiber optic endoscopy is recommended. Positron emission tomography (PET)/CT or magnetic resonance imaging scans should be performed twice a year.

\section{Discussion}

The present study describes the unusual report of an elderly male patient who suffered simultaneously from a nasopharyngeal extramedullary plasmacytoma, rich in Mott cells and in eosinophils, synchronous with an axillary lymph node small lymphocytic lymphoma with a variant paraimmunoblastic transformation. No clinical or pathological evidence of multiple myeloma, or clinical features of CLL were identified.

The lack of attention to the mild to moderate peripheral blood eosinophilia diagnosed 12 years prior to admission in the present patient may have delayed a diagnosis of chronic nasopharyngitis. To this extent, the diagnoses of allergic rhinitis, nasal polyps and aspirin hypersensitivity, individually or in combination, may have been missed. Any of these diagnoses may be associated with nasopharyngitis and are often rich in eosinophils, and anyone of these disorders may have preceded the extramedullary plasmacytoma at this unusual location (6-8).

The present patient had never previously presented with symptoms of CLL, nor were signs of this condition detected. The findings in the axillary lymph node were diagnostic of atypical SLL, however the diagnosis of a transformation into paraimmunoblastic lymphoma was favored due to the cytological features of the larger cells, mainly located in the large proliferation centers. This is an unusual form of progression of SLL, particularly since the lymphoma was diagnosed for the first time during the patients second admission. Additionally, the nasopharyngeal plasmacytoma was identified shortly prior to the lymph node biopsy, complicating the issue further. This occurrence has been described in a small number of cases (9-11). In one of these case reports, a patient with chronic lymphocytic leukemia (CLL) revealed lymphadenopathy with evidence of an extramedullary plasmacytoma. In a second case, CLL was complicated by a plasmacytoma. A molecular study demonstrated that the plasmacytoma was clonally different from the CLL. The authors of the third case considered the composite tumor as transformation of CLL into plasmacytoma. Notably, CLL/SLL with paraimmunoblastic transformation has been reported previously (12), and has been retained as a provisional entity of low-grade B-cell lymphoma (13).

An elderly patient is presented who initially developed an extramedullary nasopharyngeal plasmacytoma rich in eosinophils. Although no previous biopsy of this tissue was performed, nor were allergic rhinitis or asthma diagnosed, this may suggest chronic nasopharyngitis as the background of this plasmacytoma. The rare occurrence of a synchronous paraimmunoblastic transformation of small lymphocytic lymphoma was diagnosed two weeks subsequent to this in an axillary lymph node.

\section{Acknowledgements}

The authors would like to thank Kibbutz Sde-Boker, Israel, for hosting them during the preparation of the manuscript.

\section{References}

1. Chang YL, Chen PY and Hung SH: Extramedullary plasmacytoma of the nasopharynx: A case report and review of the literature. Oncol Lett 7: 458-460, 2014.

2. Ichimura K, Sugimura H, Naito A and Maeda Y: How to manage patients with hard to recognize postnasal drip? Rhinology 37 : 164-167, 1999.

3. Kroft SH, Dawson DB and McKenna RW: Large cell lymphoma transformation of chronic lymphocytic leukemia/small lymphocytic lymphoma. A flow cytometry analysis of seven cases. Am J ClinPathol 115: 385-395, 2001.

4. Creach KM, Foote RL, Neben-Wittich MA and Kyle RA: Radiotherapy for extramedullary plasmacytoma of the head and neck. Int J Radiat Oncol Biol Phys 73: 789-794, 2009.

5. Tournier-Rangeard L, Lapeyre M, Graff-Caillaud P, Mege A, Dolivet G, Toussaint B, Charra-Brunaud C, Hoffstetter S, Marchal C and Peiffert D: Radiotherapy for solitary extramedullary plasmacytoma in the head and neck region: A dose greater than 45 Gy to the target volume improves the local control. Int J Radiat Oncol Biol Phys 64: 1013-1017, 2006.

6. Horiguti S: Asthma and nasopharyngitis. Allerg Immunol (Leipz) 20-21: 413-425, 1975.

7. Obtulowicz K, Chlap Z and Olszewski E: Bronchial asthma and pathological processes of the nasopharynx. I Clinical analysis during remission and attack. Ann Allergy 42: 44-48, 1979.

8. Rebhun J: A new approach to the treatment of allergic nasopharyngitis. Ann Allergy 39: 175-178, 1977.

9. Chantepie SP, Cabrera Q, Mear JB, Salaun V, Lechapt-Zalcman E and Macro M: Unusual extramedullary plasmacytoma: A rare but possible cause of lymphadenopathy in chronic lymphocytic leukemia. Case Rep Med 2015: 657049, 2015.

10. Yahata N, Iwase O, Iwama H, Tauchi T, Kawanishi Y, Serizawa H and Ohyashiki K: Chronic lymphocytic leukemia complicated by plasmacytoma originating from different clones. Leuk Lymphoma 39: 203-207, 2000.

11. Pines A, Ben-Bassat I, Selzer G and Ramot B: Transformation of chronic lymphocytic leukemia to plasmacytoma. Cancer 54: 1904-1907, 1984.

12. Chuang SS, Liao YL, Liou CP, Wiggins ML, Ye H, Du MQ, Isaacson PG and Chang CC: Chronic lymphocytic leukemia with paraimmunoblastic transformation - with comparative genomic hybridization and review of the literature. Pathol Res Pract 206: 276-281, 2010.

13. Sakata S, Tsuyama N and Takeuchi K: Pathology of indolent B-cell neoplasms other than follicular lymphoma. J Clin Exp Hematop 54: 11-22, 2014. 